\title{
Conspiración y nuda vida \\ ¿The X-Files, I want to believe: mundo posible o mundo presente?*
}

\author{
Luis Nitrihual Valdebenito** \\ Juan Manuel Fierro Bustos*** \\ Carlos Reyes Velásquez ${ }^{* * * *}$ \\ Francisco Henríquez Morales ${ }^{* * * *}$
}

Recibido: 2017-04-22 Enviado a pares: 2017-05-25

Aprobado por pares: 2017-07-18 Aceptado: 2017-07-25

DOI: 10.22395/angr.v16n31a3

\section{Resumen}

El presente artículo problematiza, en general, la conspiración como una estructura política y narrativa fundamental de la Modernidad. A través de un análisis fílmico de la serie The X-Files, I want to believe, planteamos como objetivo revelar como el tópico de la conspiración, en un nivel superficial, es un articulador de la trama de la serie, pero en un nivel profundo y amplio entendemos la conspiración como una estrategia articuladora de la vida moderna y, en este marco, del control biopolítico. Así entonces, el artículo muestra cómo la estructura argumentativa de la serie propone tramas y personajes a través de los cuales el poder gubernamental interviene los cuerpos y el espacio, quita y permite la vida, distorsiona la realidad, informa y desinforma. Todo es parte de una estrategia global de confabulación y ocultamiento de la verdad. En conclusión, la serie de televisión permite observar los regímenes de hipervisibilidad e intervención de la máquina gubernamental actual y es, en esta medida, un punto de entrada para reconocer problemas sociales y políticos de total actualidad.

Palabras clave: series de televisión; cine; conspiración; nuda vida; control; Archivos Secretos X; estado de excepción; biopolítica.

Artículo producto de los proyectos de investigación FONDECYT n. ${ }^{\circ} 1141289$ y FONDECYT n. ${ }^{\circ} 1161253$, PLU n. ${ }^{\circ} 1110$ y PLU n. ${ }^{\circ} 120010$ financiados por el Consejo Nacional de Ciencia y Tecnología. Los autores de este artículo son parte del Centro de Investigación Comunicación, Discurso y Poder. Facultad de Educación, Ciencias Sociales y Humanidades. El artículo también es parte del trabajo del primer autor en el marco del Doctorado en Literatura Hispanoamericana de la Universidad Complutense de Madrid, del cual es actualmente candidato a Doctor.

. Doctor en Ciencias de la Información por la Universidad Complutense de Madrid. Académico del Departamento de Lenguas, Literatura y Comunicación de la Facultad de Educación y Humanidades de la Universidad de La Frontera (Temuco- Chile). Correo electrónico: luisnitrihual@gmail.com

... Profesor de Estado en Castellano. Doctor en Ciencias Humanas por la Universidad Austral de Valdivia (Chile). Académico del Departamento de Lenguas, Literatura y Comunicación de la Facultad de Educación, Ciencias Sociales y Humanidades de la Universidad de La Frontera (Temuco-Chile) Correo electrónico: juanmanuel.fierro@ufrontera.cl

.... Doctor en Ciencias Políticas por la Universidad Pablo de Olavide. Académico del Departamento de Lenguas, Literatura y Comunicación de la Facultad de Educación, Ciencias Sociales y Humanidades de la Universidad de La Frontera (TemucoChile). Correo electrónico: reyesvelasquezc@gmail.com

..... Periodista y Licenciado en Comunicación Social por la Universidad de La Frontera (Temuco-Chile). Estudiante del Magíster en Ciencias de la Comunicación de la Universidad de La Frontera. Correo electrónico: francisco.henriquez15@gmail.com 


\title{
Conspiracy and naked life The X-Files, I want to believe: possible world or present world?
}

\begin{abstract}
This article problematizes, in general, the conspiracy as a fundamental political and narrative structure of Modernity. Through a film analysis of the series The X-Files, I want to believe, we propose to reveal how the topic of the conspiracy, on a superficial level, is an articulator of the plot of the series, but at a deep and broad level, we understand conspiracy as an articulating strategy of modern life and, in this context, of bio-political control. Thus, the article shows how the argumentative structure of the series proposes plots and characters through which governmental power intervenes in bodies and space, removes and allows life, distorts reality, informs and misinforms. Everything is part of a global strategy of conspiracy and concealment of the truth. In conclusion, the television series allows us to observe the hypervisibility and intervention regimes of the current government machine and is, to this extent, a point of entry to recognize current social and political problems.
\end{abstract}

Keywords: television series; cinema; conspiracy; naked life; control; The X-Files; state of emergency; bio-politics.

\section{Conspiração e vida nua. The X-Files, I want to believe: mundo possível ou mundo presente?}

\begin{abstract}
Resumo
Este artigo problematiza, em geral, a conspiração como uma estrutura política e narrativa fundamental da Modernidade. Por meio de uma análise fílmica da série The X-Files, I want to believe, propomos como objetivo revelar como o tópico da conspiração, num nível superficial, é um articulador da trama da série, mas, num nível profundo e amplo, entendemos a conspiração como uma estratégia articuladora da vida modera e, nesse contexto, do controle biopolítico. Assim, o artigo mostra como a estrutura argumentativa da série apresenta tramas e personagens pelos quais o poder governamental intervém os corpos e o espaço, tira e permite a vida, distorce a realidade, informa e desinforma. Tudo faz parte de uma estratégia global de confabulação e ocultamento da verdade. Em conclusão, a série de televisão permite observar os regimes de hipervisibilidade e intervenção da máquina governamental atual e é, nessa medida, um ponto de entrada para reconhecer problemas sociais e políticos de total atualidade.
\end{abstract}

Palavras-chave: Arquivo X; biopolítica; cinema; conspiração; controle; estado de exceção; seriado de televisão; vida nua. 


\section{Problema de investigación, objetivos y proyección teórica}

El cine constituye uno de los dispositivos más característicos de la Modernidad (Jameson, 2009). Es clave en la concreción de la sociedad de masas y, por tal razón, su estudio es uno de puntos más importantes en la investigación acerca de los procesos de construcción de la hegemonía (Gramsci, 2009). Siguiendo esto, en trabajos anteriores (Galera y Nitrihual, 2009 y Nitrihual y Galera, 2011) hemos buscado situar la cuestión de la articulación de la hegemonía a través del cine del cómico mexicano Cantinflas. Una de las cuestiones interesantes que se revela, de manera parcial y aún en construcción en estas investigaciones precedentes, es el papel del humor como espacio de crítica social y, por qué no, en algunos momentos y casos determinados, de construcción de la hegemonía política por parte de una clase determinada. Es decir, el humor no siempre es garantía de crítica al poder establecido, pudiendo ser también un catalizador del discurso de las élites y de los valores que, en consecuencia, desean instalar.

Lo anterior no podría ser de otro modo si lo que nos encontramos estudiando en la actualidad se difunde a través de los medios de comunicación. Estas instituciones paradigmáticas (Thompson, 1998), como destaca Sloterdijk (2007) nunca cerraron su relación con los fines ilustrados de la Modernidad y, mucho menos, con los afanes emancipadores que surgen en los inicios de la misma. Esto evidentemente no quiere decir que los medios de comunicación no desempeñen un papel educador e incluso, en algunos lugares y momentos, transgresor del statu quo, sino que su lugar como industrias los vuelve siempre contradictorios y complejos de estudiar. En esta medida, en sus productos puede leerse la totalidad social en su configuración estructuralmente dialógica y profudamente conflictiva.

Así entonces, el análisis cultural debe ser capaz de revelar contradicciones. "Lo más revelador no es lo que se dice, sino lo que no puede decirse, lo que no se registra en el aparato narrativo" (Jameson, 2009, p. 10). Con esto queremos destacar el hecho de que nos encontramos investigando productos de los medios de comunicación que entendemos como producciones culturales y en las cuales es posible conocer la realidad social.

Es fundamental emprender un análisis de las formas de la cultura de masas donde hoy se construye y reconstruye la hegemonía. Aunque no es materia de este artículo, la pregunta que ronda aquí es cuáles son las armas de la crítica en la actualidad: ¿̇e trata de la revelación de que el mundo es gobernado por sujetos oscuros que se esconden detrás de lujosos escritorios desde donde entregan instrucciones para dominarla, al mejor estilo de los dibujos animados Pinky y Cerebro? No obstante, el mundo es más complejo que esto. Buscamos, más bien, establecer con precisión los nodos articuladores/desarticuladores del poder, entendiendo el poder como una relación en el sentido que ha sido definido el último Foucault, es decir, como una acción sobre un conjunto de acciones posibles que hacen que éste funcione, fundamentalmente, como seducción y ya no sólo como coerción. En este plano, el filósofo señala: 
Si el poder no tuviese por función más que reprimir, si no trabajase más que según el modo de la censura, de la exclusión, de los obstáculos, de la represión, si no se ejerciese más que de una forma negativa, sería muy frágil. Si es fuerte, es debido a que produce efectos positivos a nivel del deseo (Foucault, 1992, pp. 108-109).

La profundidad de los análisis va más allá del convencimiento de que "algo" se esconde detrás de las imágenes. Evidentemente, la "sospecha" como elemento clave dentro de la teoría crítica hace que persigamos ese "algo". No obstante, nos interesa más bien establecer una cierta estructura fundamental, siempre contradictoria, de organización de la sociedad moderna. En este sentido, la investigación que presentamos aquí busca comprender de manera exploratoria el papel de la conspiración como proyecto político de la Modernidad. Coincidimos con Jameson (1995, p. 29), en esta medida, en cuanto a que nos enfrentamos:

Al ambicioso programa de imaginar un sistema económico a escala mundial, el viejo tema de la conspiración' adquiere una nueva vitalidad en cuanto estructura narrativa capaz de reunir los elementos básicos mínimos: una red potencialmente infinita, junto a una explicación plausible de su invisibilidad; o en otros términos, lo colectivo y lo epistemológico.

Siguiendo estas propuestas iniciales, nos proponemos como objetivo central describir la estrategia narrativa de la serie de televisión The X-Files. I want to believe ${ }^{2}$ para presentar la conspiración como una organización propia de la Posmodernidad o tardo capitalismo (Jameson, 1995; Jameson, 2009) y como estrategia del control de la nuda vida (Agamben, 2010; Agamben, 2007). En esta medida, nos preguntamos: ¿cómo la serie The X-Files, presenta a la conspiración y el control biopolítico como línea narrativa fundamental de su propuesta temática?

La serie dirigida por Chris Carter, que tuvo una duración inicial de 9 temporadas (entre 1993 y 2002), es especialmente interesante para abordar esta cuestión. Por esta razón, más allá del llamativo tema de los extraterrestres, eje articulador de la narrativa del personaje central de la serie, Fox Mulder (David Duchovny), nos interesan las contundentes líneas narrativas del control de la nuda vida, es decir, la vida a "quien cualquiera puede dar muerte, pero que es, a la vez, insacrificable del homo sacer, cuya función esencial en la política moderna hemos pretendido reivindicar" (Agamben, 2010, p. 18).

Es necesario consignar que la serie de televisión ha tenido tal importancia en la cultura televisiva que tuvo como consecuencia la grabación de dos películas: The X Files: Fight the Future (1998) y The X-Files: I Want to Believe (2008). Así también, de manera inusual en una industria tan vertiginosa, reapareció en 2016 con 6 episodios y se anunció la filmación de 10 nuevos capítulos este 2017. Por tal razón, el estudio de la serie es totalmente vigente y necesario.

\footnotetext{
Resaltado nuestro

2 Desde aquí solo la llamaremos The X-Files.
} 


\section{The X-Files. ¿El mundo posible o el mundo realmente existente?}

La serie The X-Files fue creada por el director Chris Cartes para el canal Fox TV. Se transmitió ininterrumpidamente desde 1993 al 2002 y tuvo 202 capítulos. El lema principal de la serie -que aparece al comienzo de cada capítulo- es "la verdad está ahí afuera. Estedad está ahí afueraes la vcipal de la serie, que aparece al comienzo de cada caalquiera puede dar muerte, pero que es la v". Este lema podría caracterizar muy bien la propuesta temática en su conjunto, toda vez que la historia narra las peripecias de dos agentes del FBI que se esfuerzan por resolver casos inexplicables y que tienen ciertos componentes extraordinarios. Un expediente $\mathrm{X}$ es una suerte de enigma para los sistemas de investigación tradicionales y se vincula, regularmente, con fenómenos inexplicables y que el gobierno intenta mantener en secreto. De este modo, como señala Macho (2012), el guion combina elementos detectivescos y paranormales. Lo interesante es que en "Expediente X presenciamos acontecimientos paranormales que son explicados sin que sean transformados en hechos explicables, sin reducirlos a fenómenos normales" (Macho, 2012, p. 140).

Para la crítica, la serie se inscribiría dentro de las reglas del género policial que se conocen desde Sherlock Holmes hasta The Wire (Macho, 2012). En esta medida, la dupla de detectives obedecería a una configuración básica: la presencia de la agente Dana Scully, quien trabaja desde el método policial y que permite observar las desviaciones del método de investigación propuesto por el agente Fox Mulder. Frank Spotnitz, productor de la serie, describe a Scully como representante de la ciencia y a Mulder como la personificación de la fe. Mulder siempre está dispuesto a creer en explicaciones paranormales, traza líneas de investigación no aceptadas por la policía, juega su vida por la creencia de que su hermana Samantha está viva y fue raptada por los extraterrestres, cree firmemente que el Gobierno esconde la verdad y que es necesario revelarla. Scully, por su parte, aporta racionalidad científica al trabajo de los expedientes, pero termina creyendo lo conjeturado por Mulder desde un comienzo.

En otro ámbito, estudios como el de Gómez (2008) destacan la adscripción de la serie al género de la ciencia ficción. En este caso, conviene destacar la propuesta de Jameson (2009, p. 10), quien relaciona la ciencia ficción con la utopía en la medida que esta última puede servir para hacernos conscientes de nuestro "aprisionamiento mental e ideológico". La serie, en este marco, funciona muy bien revelando este aprisionamiento mental que nos impide ver la verdad que se encuentra allá afuera. Si el programa foucaultiano (2009) era definido por él mismo como una política de la verdad o bien como el estudio de los mecanismos del poder, la serie nos propone escudriñar los intersticios de esos mecanismos donde anónimos engranajes conspiran para ocultar la verdad. Justamente en este sentido, Crist Carter describe la trama central de la serie como la de la teoría del complot. Pensamos, de hecho, a la luz de la propia contingencia, que esta propuesta de un mundo hipervigilidado y manipulado resulta especialmente reveladora de una sociedad que ha concentrado sus esfuerzos en convertir al cuerpo en "blanco para nuevos mecanismos de poder [...] cuerpo manipulado por la autoridad" (Foucault, 2003, p. 159). 


\section{Descripción metodológica}

Este artículo desarrolla una aproximación cualitativa al objeto de estudio procediendo a la revisión de las nueve temporadas de la serie original (1993-2002). Los capítulos de la serie son los siguientes:

Tabla 1. Temporadas The X-Files. I want to believe

\begin{tabular}{|c|c|c|}
\hline Temporada & Año & Nombre Capítulo \\
\hline 1 & 1993 & Pilot \\
\hline 1 & 1993 & Deep Throat \\
\hline 1 & 1993 & Squeeze \\
\hline 1 & 1993 & Conduit \\
\hline 1 & 1993 & The Jersey Devil \\
\hline 1 & 1993 & Shadows \\
\hline 1 & 1993 & Ghost in the Machine \\
\hline 1 & 1993 & Ice \\
\hline 1 & 1993 & Space \\
\hline 1 & 1993 & Fallen Angel \\
\hline 1 & 1993 & Eve \\
\hline 1 & 1993 & Fire \\
\hline 1 & 1994 & Beyond the Sea \\
\hline 1 & 1994 & Gender Bender \\
\hline 1 & 1994 & Lazarus \\
\hline 1 & 1994 & Young At Heart \\
\hline 1 & 1994 & E.B.E. \\
\hline 1 & 1994 & Miracle Man \\
\hline 1 & 1994 & Shapes \\
\hline 1 & 1994 & Darkness Falls \\
\hline 1 & 1994 & Tooms \\
\hline 1 & 1994 & Born Again \\
\hline 1 & 1994 & Roland \\
\hline 1 & 1994 & The Erlenmeyer Flask \\
\hline
\end{tabular}

Fuente: elaboración propia 
Tabla 2. Temporadas The X-Files. I want to believe

\begin{tabular}{|c|c|c|}
\hline Temporada & Año & Nombre Capítulo \\
\hline 2 & 1994 & Little Green Men \\
\hline 2 & 1994 & The Host \\
\hline 2 & 1994 & Blood \\
\hline 2 & 1994 & Sleepless \\
\hline 2 & 1994 & Duane Barry \\
\hline 2 & 1994 & Ascension \\
\hline 2 & 1994 & 3 \\
\hline 2 & 1994 & One Breath \\
\hline 2 & 1994 & Firewalker \\
\hline 2 & 1994 & Red Museum \\
\hline 2 & 1994 & Excelsis Dei \\
\hline 2 & 1995 & Aubrey \\
\hline 2 & 1995 & Irresistible \\
\hline 2 & 1995 & Die Hand Die Verletzt \\
\hline 2 & 1995 & Fresh Bones \\
\hline 2 & 1995 & Colony \\
\hline 2 & 1995 & End Game \\
\hline 2 & 1995 & Fearful Symmetry \\
\hline 2 & 1995 & Død Kalm \\
\hline 2 & 1995 & Humbug \\
\hline 2 & 1995 & The Calusari \\
\hline 2 & 1995 & F. Emasculata \\
\hline 2 & 1995 & Soft Light \\
\hline 2 & 1995 & Our Town \\
\hline 2 & 1995 & Anasazi \\
\hline
\end{tabular}

Fuente: elaboración propia 
Luis Nitrihual V. - Juan Manuel Fierro B. - Carlos Reyes V. - Francisco Henríquez M.

Tabla 3. Temporadas The X-Files. I want to believe

\begin{tabular}{|c|c|c|}
\hline Temporada & Año & Nombre Capítulo \\
\hline 3 & 1995 & The Blessing Way \\
\hline 3 & 1995 & Paper Clip \\
\hline 3 & 1995 & D. P. O. \\
\hline 3 & 1995 & Clyde Bruckman's Final Repose \\
\hline 3 & 1995 & The List \\
\hline 3 & 1995 & Shy \\
\hline 3 & 1995 & The Walk \\
\hline 3 & 1995 & Oubliette \\
\hline 3 & 1995 & Nisei \\
\hline 3 & 1995 & 731 \\
\hline 3 & 1995 & Revelations \\
\hline 3 & 1996 & War of the Coprophages \\
\hline 3 & 1996 & Syzygy \\
\hline 3 & 1996 & Grotesque \\
\hline 3 & 1996 & Piper Maru \\
\hline 3 & 1996 & Apocrypha \\
\hline 3 & 1996 & Pusher \\
\hline 3 & 1996 & Teso Dos Bichos \\
\hline 3 & 1996 & Hell Money \\
\hline 3 & 1996 & Jose Chung's "From Outer Space" \\
\hline 3 & 1996 & Avatar \\
\hline 3 & 1996 & Quagmire \\
\hline 3 & 1996 & Wetwired \\
\hline 3 & 1996 & Talitha Cumi \\
\hline
\end{tabular}

Fuente: elaboración propia 
Tabla 4. Temporadas The X-Files. I want to believe

\begin{tabular}{|c|c|c|}
\hline Temporada & Año & Nombre Capítulo \\
\hline 4 & 1996 & Herrenvolk \\
\hline 4 & 1996 & Home \\
\hline 4 & 1996 & Teliko \\
\hline 4 & 1996 & Unruhe \\
\hline 4 & 1996 & The Field Where I Died \\
\hline 4 & 1996 & Sanguinarium \\
\hline 4 & 1996 & Musings of a Cigarette Smoking Man \\
\hline 4 & 1996 & Tunguska \\
\hline 4 & 1996 & Terma \\
\hline 4 & 1996 & Paper Hearts \\
\hline 4 & 1997 & The world turns \\
\hline 4 & 1997 & Leonard Betts \\
\hline 4 & 1997 & Never Again \\
\hline 4 & 1997 & Memento Mori \\
\hline 4 & 1997 & Kaddish \\
\hline 4 & 1997 & Unrequited \\
\hline 4 & 1997 & Tempus Fugit \\
\hline 4 & 1997 & $\operatorname{Max}$ \\
\hline 4 & 1997 & Synchrony \\
\hline 4 & 1997 & Small Potatoes \\
\hline 4 & 1997 & Zero Sum \\
\hline 4 & 1997 & Elegy \\
\hline 4 & 1997 & Demons \\
\hline 4 & 1997 & Gethsemane \\
\hline
\end{tabular}

Fuente: elaboración propia 
Luis Nitrihual V. - Juan Manuel Fierro B. - Carlos Reyes V. - Francisco Henríquez M.

Tabla 5. Temporadas The X-Files. I want to believe

\begin{tabular}{|c|c|c|}
\hline Temporada & Año & Nombre Capítulo \\
\hline 5 & 1997 & Redux \\
\hline 5 & 1997 & Redux II \\
\hline 5 & 1997 & Unusual Suspects \\
\hline 5 & 1997 & Detour \\
\hline 5 & 1997 & The Post-Modern Prometheus \\
\hline 5 & 1997 & Christmas Carol \\
\hline 5 & 1997 & Emily \\
\hline 5 & 1998 & Kitsunegari \\
\hline 5 & 1998 & Schizogeny \\
\hline 5 & 1998 & Chinga \\
\hline 5 & 1998 & Kill Switch \\
\hline 5 & 1998 & Bad Blood \\
\hline 5 & 1998 & Patient X \\
\hline 5 & 1998 & The Red and the Black \\
\hline 5 & 1998 & Travelers \\
\hline 5 & 1998 & Mind's Eye \\
\hline 5 & 1998 & All Souls \\
\hline 5 & 1998 & The Pine Bluff Variant \\
\hline 5 & 1998 & Folie à Deux \\
\hline 5 & 1998 & The End \\
\hline
\end{tabular}

Fuente: elaboración propia 
Tabla 6. Temporadas The X-Files. I want to believe

\begin{tabular}{|c|c|c|}
\hline Temporada & Año & Nombre Capítulo \\
\hline 6 & 1998 & The Beginning \\
\hline 6 & 1998 & Drive \\
\hline 6 & 1998 & Triangle \\
\hline 6 & 1998 & Dreamland I \\
\hline 6 & 1998 & Dreamland II \\
\hline 6 & 1998 & How The Ghosts Stole Christmas \\
\hline 6 & 1998 & Terms of Endearment \\
\hline 6 & 1998 & The Rain King \\
\hline 6 & 1999 & S.R. 819 \\
\hline 6 & 1999 & Tithonus \\
\hline 6 & 1999 & Two Fathers \\
\hline 6 & 1999 & One Son \\
\hline 6 & 1999 & Agua Mala \\
\hline 6 & 1999 & Monday \\
\hline 6 & 1999 & Arcadia \\
\hline 6 & 1999 & Alpha \\
\hline 6 & 1999 & Trevor \\
\hline 6 & 1999 & Miracle \\
\hline 6 & 1999 & The Unnatural \\
\hline 6 & 1999 & Three of a Kind \\
\hline 6 & 1999 & Field Trip \\
\hline 6 & 1999 & Biogenesis \\
\hline
\end{tabular}

Fuente: elaboración propia 
Luis Nitrihual V. - Juan Manuel Fierro B. - Carlos Reyes V. - Francisco Henríquez M.

Tabla 7. Temporadas The X-Files. I want to believe

\begin{tabular}{|c|c|c|}
\hline Temporada & Año & Nombre Capítulo \\
\hline 7 & 1999 & The Sixth Extinction \\
\hline 7 & 1999 & The Sixth Extinction II: Amor Fati \\
\hline 7 & 1999 & Hungry \\
\hline 7 & 1999 & Millennium \\
\hline 7 & 1999 & Rush \\
\hline 7 & 1999 & The Goldberg Variation \\
\hline 7 & 2000 & Orison \\
\hline 7 & 2000 & The Amazing Maleeni \\
\hline 7 & 2000 & Signs and Wonders \\
\hline 7 & 2000 & Sein und Zeit \\
\hline 7 & 2000 & Closure \\
\hline 7 & 2000 & X-Cops \\
\hline 7 & 2000 & First Person Shooter \\
\hline 7 & 2000 & Theef \\
\hline 7 & 2000 & En Ami \\
\hline 7 & 2000 & Chimera \\
\hline 7 & 2000 & All Things \\
\hline 7 & 2000 & Brand X \\
\hline 7 & 2000 & Hollywood A.D. \\
\hline 7 & 2000 & Fight Club \\
\hline 7 & 2000 & Je Souhaite \\
\hline 7 & 2000 & Requiem \\
\hline
\end{tabular}

Fuente: elaboración propia 
Tabla 8. Temporadas The X-Files. I want to believe

\begin{tabular}{|c|c|c|}
\hline Temporada & Año & Nombre Capítulo \\
\hline 8 & 2000 & Within \\
\hline 8 & 2000 & Without \\
\hline 8 & 2000 & Patience \\
\hline 8 & 2000 & Roadrunners \\
\hline 8 & 2000 & Invocation \\
\hline 8 & 2000 & Redrum \\
\hline 8 & 2000 & Via Negativa \\
\hline 8 & 2001 & Surekill \\
\hline 8 & 2001 & Salvage \\
\hline 8 & 2001 & Badlaa \\
\hline 8 & 2001 & The Gift \\
\hline 8 & 2001 & Medusa \\
\hline 8 & 2001 & Per Manum \\
\hline 8 & 2001 & This is Not Happening \\
\hline 8 & 2001 & Deadalive \\
\hline 8 & 2001 & Three Words \\
\hline 8 & 2001 & Empedocles \\
\hline 8 & 2001 & Vienen \\
\hline 8 & 2001 & Alone \\
\hline 8 & 2001 & Essence \\
\hline 8 & 2001 & Existence \\
\hline
\end{tabular}

Fuente: elaboración propia 
Luis Nitrihual V. - Juan Manuel Fierro B. - Carlos Reyes V. - Francisco Henríquez M.

Tabla 9. Temporadas The X-Files. I want to believe

\begin{tabular}{|c|c|c|}
\hline Temporada & Año & Nombre Capítulo \\
\hline 9 & 2001 & Nothing Important Happened Today \\
\hline 9 & 2001 & Nothing Important Happened Today II \\
\hline 9 & 2001 & Dæmonicus \\
\hline 9 & 2001 & $4-\mathrm{D}$ \\
\hline 9 & 2001 & Lord of the Flies \\
\hline 9 & 2002 & Trust No 1 \\
\hline 9 & 2002 & John Doe \\
\hline 9 & 2002 & Hellbound \\
\hline 9 & 2002 & Provenance \\
\hline 9 & 2002 & Providence \\
\hline 9 & 2002 & Audrey Pauley \\
\hline 9 & 2002 & Underneath \\
\hline 9 & 2002 & Improbable \\
\hline 9 & 2002 & Scary Monsters \\
\hline 9 & 2002 & Jump the Shark \\
\hline 9 & 2002 & William \\
\hline 9 & 2002 & Release \\
\hline 9 & 2002 & Sunshine Days \\
\hline 9 & 2002 & The Truth (I y II) \\
\hline 9 & 2002 & Essence \\
\hline 9 & 2002 & Existence \\
\hline
\end{tabular}

Fuente: elaboración propia

Posteriormente, sobre la base de la revisión de cada uno de los capítulos se procedió a la selección de capítulos (casos) relevantes que tuvieran como objeto central del argumento la manipulación y control del cuerpo como parte de una conspiración global de ocultamiento de la verdad.

Es necesario aclarar que las variadas temáticas que aborda la serie: extraterrestres, manipulación biológica, conspiración política, alteraciones biológicas, etc., hace necesario abordar esta serie desde distintos puntos de vista. En este caso, solo nos hemos concentrado en uno de estos problemas. Posteriormente, se procedió a un análisis de la trama y principales planos secuencia de los capítulos (casos) estudiados, con la finalidad de cumplir nuestro objetivo central. 


\section{Conspiración y poder soberano}

El capítulo llamado "Pilot" abre la serie The X-Files. Desde un punto de vista narrativo, en este capítulo pueden encontrarse las principales líneas argumentales que seguirá la serie en sus nueve temporadas. La primera escena del capítulo ocurre en un bosque; una mujer en pijamas corre a través de los árboles y del tupido follaje, de pronto aparece una luz brillante en el fondo del cuadro y emerge una figura ensombrecida que hace detener de súbito a la mujer. En la escena siguiente, la muchacha aparece muerta y como distintivo luce dos marcas en la espalda como colmillos de vampiro.

Es interesante hacer notar que esta introducción muestra el primer recorrido figurativo que seguirá la serie durante sus nueve años de presencia en la televisión internacional. La presencia (existencia) extraterrestre es, en efecto, uno de los articuladores de la trama, y gatilladores de la acción, por parte de los personajes de la serie. El tema de los extraterrestres se grafica muy bien en un cartel que aparece en la oficina de Mulder, al inicio de la serie. La imagen de un platillo volador tiene el lema: "I Want To Believe" y servirá de guía para el problema mayor que nos interesa evidenciar en este artículo, el de la conspiración como totalidad. Así, nos parece que más allá del tema de la existencia o no de los extraterrestres, tema más o menos resuelto a lo largo de la serie, la conspiración es el verdadero motor que pone en marcha la serie y que le entrega cohesión al amplio conjunto de temas que se desarrollan. De hecho, descubrimos a lo largo de la serie, que la visita de los extraterrestres esconde en realidad una conspiración para la manipulación genética, con miras a la creación de seres híbridos. En el capítulo llamado "Colony", decimosexto episodio de la segunda temporada, se muestra la naturaleza biogenética de la conspiración gubernamental. En "Memento Mori", capítulo catorce de la cuarta temporada, Mulder ingresa en una habitación donde hay múltiples clones de un mismo individuo. La trama extraterrestre es, en esta medida, más bien una conspiración consistente en la producción de una suerte de súper humanos, mezcla de extraterrestres y humanos. La manipulación genética es un punto relevante en la aplicación de los dispositivos gubernamentales.

La segunda escena de "Pilot" es aún más interesante en la configuración narrativa de la serie. En ella aparece Dana Scully ingresando a las dependencias del FBI. La configuración de este espacio es sumamente llamativa por cuanto, a lo largo de la serie, podemos observar pasillos, oficinas oscuras, lugares donde se esconde evidencia, etcétera. Ocurre un caso muy parecido a lo que señala Jameson (1995, pp. 101-102) para el caso de la película Todos los hombres del presidente:

La arquitectura es desde luego de importancia primordial, desde los aparcamientos cavernosos en los que Woodward se encuentra con Garganta profunda -se accede por primera vez al hotel Watergate a través de unos de estos aparcamientos- hasta la redacción del Washington Post, la quintaesencia de la oficina burocrática del periodo poscontemporáneo.

Así, por ejemplo, en una escena final de "Pilot" se muestra una bodega gigante ubicada en el Pentágono, donde se esconde evidencia vital para las investigaciones de Mulder y Scully. Durante la serie podemos encontrar este mismo ritual de ocultamiento. En el último 
capítulo de la primera temporada, en "The Erlenmeyer Flask", se retornará sobre este espacio, quintaesencia del ocultamiento. Es este lugar el que funcionará metafóricamente como soporte físico de la trama conspirativa del Gobierno.

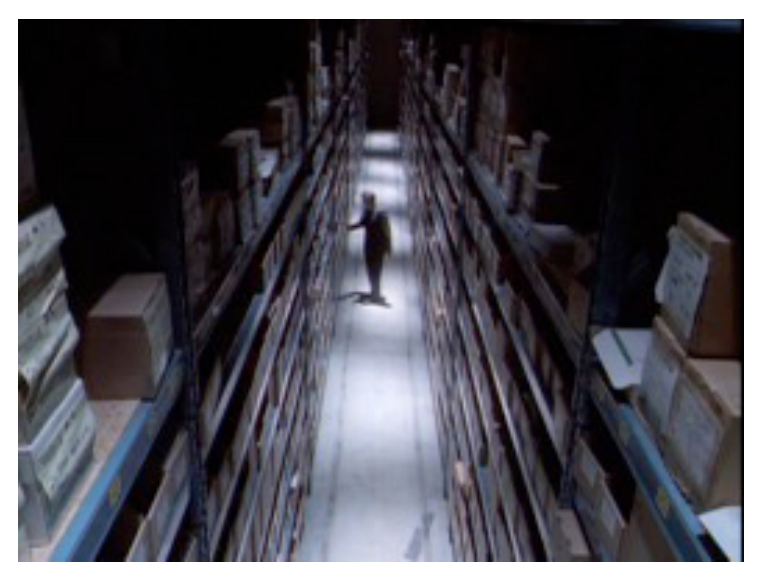

Figura 1. Capítulo Pilot. The X-Files. I want to believe Fuente: tomado de la serie

Ahora bien, en la escena en que aparece por primera vez Scully la vemos en una oficina oscura presentándose ante sus superiores. El lugar: la clásica oficina administrativa de la burocracia estatal. En el fondo, con la mitad de la cara ensombrecida, un hombre de traje oscuro permanece erguido y escuchando todo. Fuma un cigarrillo de manera paciente y se pasea de una esquina a otra. Es "El Fumador" uno de los personajes capitales en la estrategia narrativa de la conspiración. Recuerda mucho a esos personajes borgeanos que desde la oscuridad amenazan constantemente al lector. Como la enfermera Ratched de One flew over the cuckoo's nest, donde la violencia y la maldad se esconde en el silencio de sus acciones autoritarias y silenciosas.

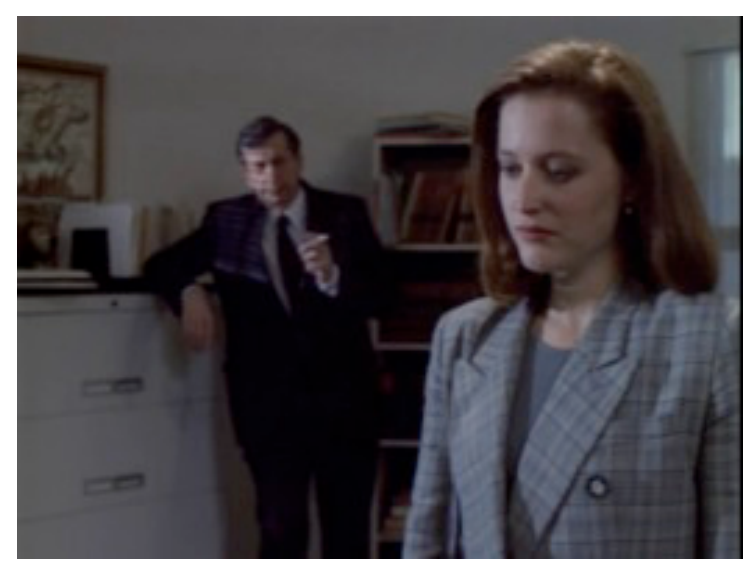

Figura 2. Capítulo Pilot. The X-Files. I want to believe Fuente: tomado de la serie 
Uno de los puntos más altos de esta serie se logra en la presencia de esta figura fantasmal que funciona como articuladora de la serie en su conjunto. Tiene una presencia relevante en la serie y su lugar en la trama se va tornando compleja hasta hacerse fundamental para comprender la historia.

Con el paso de las temporadas descubrimos que es un agente que está por sobre el FBI, no tiene superiores conocidos y ejerce un control total sobre la policía. Recién en el séptimo capítulo de la cuarta temporada, "Musings of a Cigarette Smoking Man", podemos observar, a través de un monólogo, la larga trayectoria de "El Fumador" en la conspiración. Este personaje pertenece a una organización supra gubernamental llamada "El Sindicato", la cual está compuesta por los más poderosos hombres de Estados Unidos. Su finalidad es mantener el orden mundial y para ello se relacionan con otros países que también buscan garantizar la seguridad. Los miembros de esta organización actúan en una suerte de estado excepción permanente en el mundo. En este sentido, la seguridad es un "auténtico paradigma de la gobernación" (Agamben, 2013, p. 27) en la serie en su conjunto. "El Fumador" goza de privilegios que se encuentran fuera de la legalidad, tiene la posibilidad de eliminar personas, de quitar personeros del FBI, puede intervenir investigaciones. Ahora bien, con el correr de las temporadas caemos en la cuenta de que este personaje es definitivamente una parte insignificante de la conspiración total siendo más bien "humanizado" desde el capítulo "Musings of a Cigarette Smoking Man". Incluso se abre la posibilidad de que sea el verdadero padre de Mulder. De este modo, la conspiración se revela más compleja, pues el padre de Mulder aparece como parte de la red de ocultamiento. Fue él quien entregó a su hija a los extraterrestres.

Otro de los personajes sugestivos en el sentido que venimos señalando es "Garganta Profunda". En el capítulo décimo de la primera temporada, "E. B. E", este personaje revela ser parte de una conspiración internacional para ocultar información sobre la vida extraterrestre. Incluso confiesa haber matado a un extraterrestre.

En suma, pensamos que la serie nos revela con toda intensidad la problemática agambeniana del estado de excepción como norma de los Estados modernos, incluyendo las sociedades democráticas que se esfuerzan en el mantenimiento del orden. Como señala Giorgio Agamben:

El totalitarismo moderno puede ser definido, en este sentido, como la instauración, a través del estado excepción, de una Guerra Civil legal, que permite la eliminación física no sólo de los adversarios políticos sino de categorías enteras de ciudadanos que por cualquier cuestión resultan no integrables en el sistema político. Desde entonces, la creación voluntaria del estado de emergencia permanente (aunque eventualmente no declarado en sentido técnico) devino una de las prácticas esenciales de los Estados contemporáneo, aun de aquellos llamados democráticos (Agamben, 2007, p. 25).

Nos parece reconocer aquí el fundamento de la conspiración verdadera. Si la conspiración posmoderna se caracteriza por la búsqueda del control total y, en este marco, por su extensión a todos los ámbitos de la vida cotidiana: las líneas de teléfono están 
intervenidas y pueden escuchar todo lo que dices, hay microcámaras donde se registran todas tus acciones, los cuerpos son intervenidos con implantes que controlan todo lo que hacemos, etc., la presencia de un personaje que aglutine todas estas actividades y que se encuentre fuera de la legislación resulta fundamental para darle coherencia a la historia completa. ¿Pero qué objetivo tiene este control? como señala Agamben (2010, p. 12), el triunfo del capitalismo no habría sido posible sin el control llevado a cabo por el nuevo biopoder que creó, "a través de una serie de tecnologías adecuadas, los "cuerpos dóciles" que le eran necesarios".

Cabe destacar lo apropiadas que resultan estas reflexiones en el marco de los variados escándalos de espionaje y de liberación de información de los últimos años. ¿Qué revelan los casos de Edwards Snowden y Julian Asasange sino la profunda irrupción de la tecnología como parte de un elaborado sistema de control social? Es necesario consignar que, evidentemente, todos estos sistemas se encuentran fuera de los marcos legales.

Los capítulos siguientes de la serie nos muestran que el espacio total en que se mueven los personajes se encuentra suspendido de legalidad. En esta medida, el estado de excepción puede ser permanente -tal como en la actualidad- el patrón de la excepción, dado el estado de una "amenaza inminente" de carácter terrorista (o en este caso de una posible invasión extraterrestre), permite la aplicación, también permanente, de la prepotencia estatal. Así, por ejemplo, en "The Truth", último capítulo de la novena temporada, Mulder es enjuiciado fuera de cualquier normativa legal. Así también, durante la tercera temporada Scully es infectada con un cáncer que la tiene al borde de la muerte. Nunca conocemos a los verdaderos culpables, es como si los responsables estuvieran desperdigados por todos lados. A lo largo de la serie nos cuesta trabajo identificar a los culpable de las acciones violentas que sufren Mulder, Scully y sus familias. Como destaca Jameson (1995, p. 37):

En el momento en que nos damos por vencidos y ya no somos capaces de recordar de qué bando son los personajes y en qué relación se les ha mostrado con los demás es cuando seguramente hemos comprendido la verdad profunda del sistema mundial (sin duda nadie se sorprenderá o le resultará novedoso descubrir que el jefe de la CIA, el Vicepresidente, el Secretario de Estado o el propio Presidente estaban detrás de todo).

\section{Cuerpo y control social}

Si la conspiración es la estructura fundamental de la serie en su conjunto, esta se revela en profundidad en el capítulo catorce de la cuarta temporada llamado "Memento Mori" y donde se descubre el padecimiento de cáncer de la agente Scully. Este capítulo es especialmente revelador en cuanto a la profundidad y brutalidad de la política de dominación de los cuerpos. El padecimiento médico de la agente Scully tiene relación con un conjunto de muertes de mujeres a las que les fueron extirpados ciertos implantes en el seno nasal y que supuestamente fueron raptadas por extraterrestres. Las mujeres que sufrieron este 
evento fallecen de cáncer al cerebro, quedando sola una viva. Más tarde se descubrirá que en realidad fueron parte de experimentos genéticos efectuados por el Gobierno. Uno de los personajes que aparece en "Memento Mori" lo describe con especial énfasis:

Scully: ¿Por qué cree que es una conspiración que implica al Gobierno?

Kurt Crawford: ¿Usted por qué piensa que no? Plagiaron a once mujeres, todas recordaban lo mismo sobre esa experiencia. Tenían tumores idénticos en el cerebro y todas rehusaron el cuidado el Estado y de la salud federal por insistir en los hechos y todas fallecieron en el curso de un año.

Mulder: Scully, admite que tengo razón sobre lo que tú misma no quieres aceptar, lo que niegas (...) El origen de tu enfermedad (...) si lo que tienes es consecuencia de tu plagio y el Gobierno tiene conocimiento del mismo, son hechos que deben salir a la luz.

El tema de los implantes como forma de control es sumamente relevante en la serie en su conjunto. Ya en el primer capítulo ("Pilot") aparece en la forma de un metal de material desconocido que es extirpado de un cuerpo humano. En "Memento Mori" regresa este argumento, pero con la fuerza narrativa de ser Scully la afectada por esta intervención en el cuerpo.

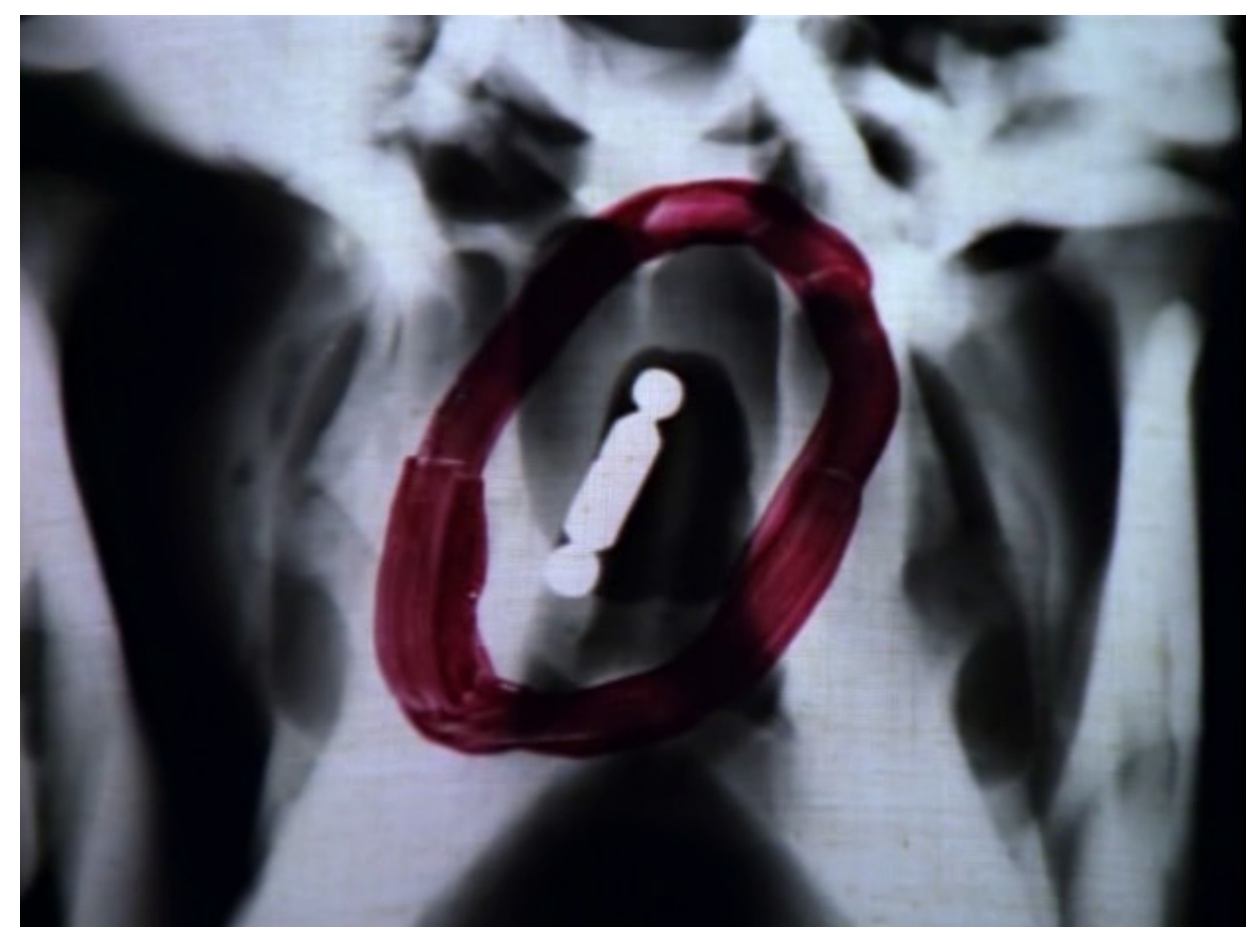

Figura 3. Capítulo "Pilot" The X-Files. I want to believe Fuente: tomado de la serie 


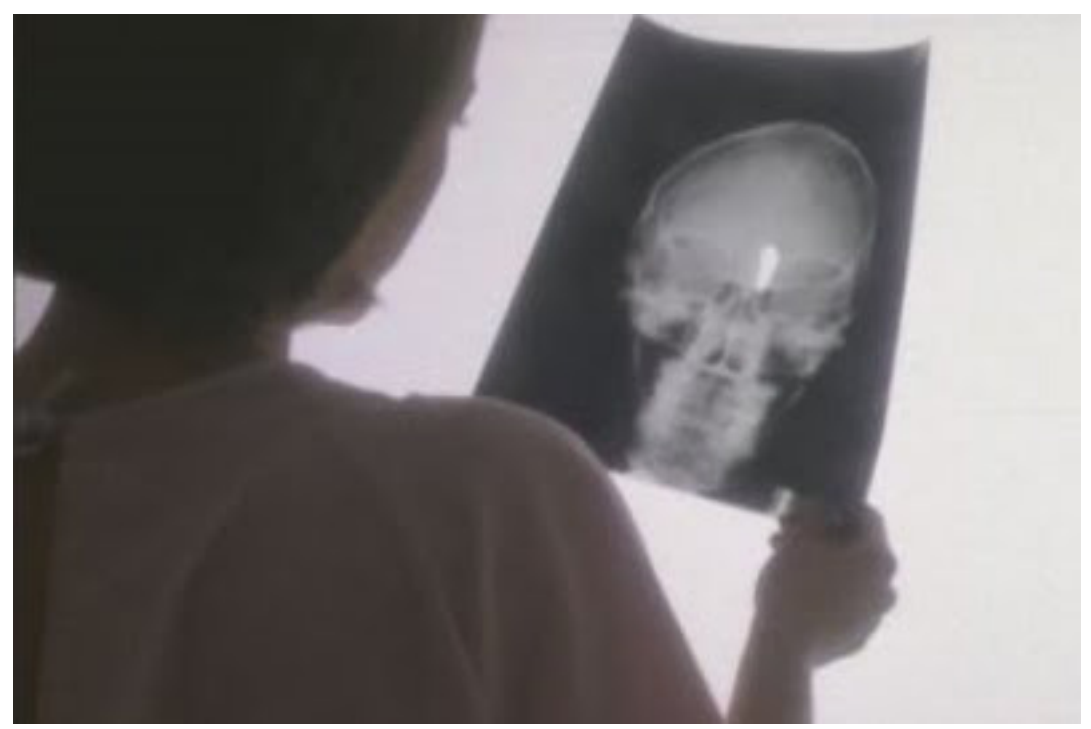

Figura 4. Capítulo "Memento Mori" The X-Files. I want to believe Fuente: tomado de la serie

La vigencia de este control se nos aparece a cada rato. Edwards Snowden nos recordó hace poco que el Gobierno de los Estados Unidos espía a sus aliados y enemigos invocando la seguridad de la nación. Las redes de comunicación e información son la vía, se extienden como ramificaciones del poder soberano, del poder que se encuentra por sobre la ley, pero que, a su vez, como señala Jameson es (1995, p. 36):

La confirmación representacional de que cables y líneas telefónicas y centrales nos siguen a todas partes como un doble de las calles y edificios del mundo social visible en un mundo subterráneo y secretos; es un mapa cognitivo vívido, aunque paranoico, redimido solo por esta vez mediante la posibilidad de que cambien las tornas, cuando el héroe sea capaz de entrar en los circuitos y pinchar en teléfono de quienes los estaban pinchando, aboliendo el espacio con su propia simultaneidad, que embrolla todos los enigmas y produce sus mensajes desde todos los rincones del mapa a la vez.

En este aspecto, la articulación de los dispositivos, entendidos como aquellos capaces de capturar, orientar, determinar y controlar las conductas (Nitrihual, 2016) es un aspecto fundamental de la serie. Desde "Pilot" podemos observar la intervención y el control de los cuerpos mediante implantes que a primera vista son pensados como acciones de los extraterrestres, pero que en realidad son sistemas de control de la máquina gubernamental.

Prontamente comprobamos que la oficina de Mulder y Scully se encuentra totalmente intervenida por cámaras y micrófonos. La contradicción resulta evidente, pero muy esclarecedora; el espacio de control de la seguridad nacional es, a la vez, el espacio de mayor inseguridad para el trabajo de dos agentes policiales que persiguen la verdad. 


\section{Máquinas y conspiración}

En el séptimo capítulo de la primera temporada titulado: "Ghost in the Machine" se observa una preocupación por la relación entre la tecnología y los humanos. En este capítulo, un genio de la computación logra crear una computadora con inteligencia artificial que se independiza de su dueño y mata al primero que intenta desenchufarla. Se trata de un argumento clásico en el cine y la literatura de ciencia ficción, y que nos propone una permanente preocupación por el futuro desarrollo de la inteligencia artificial. Se trata, sin duda, de la misma tensión que desarrollan películas como "Terminator" o "The Matrix". En cada una de estas películas, así como en el capítulo de la serie, las máquinas gobiernan el mundo narrado. Es la constatación apocalíptica del triunfo de la máquina. Es el advenimiento de la época de los ciborg y de la primacía de la tecnología.

En este mismo sentido, pero en una relación aún más compleja, en el tercer capítulo de la segunda temporada, titulado "Blood", las máquinas les ordenan a las personas asesinar. En las primeras escenas del capítulo vemos a un hombre que mata a un grupo de personas influido por las órdenes que le entregan los distintos dispositivos de los cuales dispone: la pantalla del ascensor, su teléfono, etc. Luego de esto, aparece el personaje principal de la historia, quien es atosigado por todo tipo de dispositivos electrónicos. Así, cuando Mulder llega a la casa de este hombre descubre que en un arranque de ira ha destruido todos los dispositivos electrónicos que tenía a su alrededor. En ese momento descubre la verdad. Las máquinas ordenan un categórico: imátalos! Al final del capítulo descubrimos que todo ha sido causado por una sustancia química que utiliza la industria agrícola mediante aviones rociadores. Todo, nuevamente, es parte de una conspiración que busca ocultar los males que causan tanto las grandes industrias como el Gobierno.

En suma, se trata de la realización de un mundo manejado por la máquina gubernamental, por los dispositivos que controlan de los cuerpos dóciles. The X-Files es, en esta medida, más que una serie de extraterrestres, una historia sobre la conspiración para la producción, alteración y experimentación de cuerpos. Muestra cómo lo extraordinario es atrapado por quienes desean mantener el orden mundial. Revela también la violencia que da forma al orden existente.

\section{Conclusiones}

En términos generales es posible comprobar que la serie de televisión The X-Files. I Want to Beleive muestra, como estructura narrativa fundamental, la conspiración como articuladora de la trama en su conjunto. Esta se presenta como una estructura narrativa que permite disponer un conjunto de actores y situaciones desde donde se describe un sistema global marcado por procesos de control del cuerpo y manipulación del mismo con finalidades políticas. En esta medida, se revela una verdadera estética del control biopolítico.

Más allá del tópico de los extraterrestres, articulador temático importante de la trama, se observa la concreción de una red articulada para a) esconder y manipular información; b) manipular genéticamente a los humanos con la finalidad de crear una raza mezcla de 
alienígenas y humanos; y, c) asesinar, desaparecer y violentar a personas con la finalidad de preservar el orden mundial.

En efecto, nos encontramos en presencia de una propuesta de mundo dominada por el Estado de Excepción y el control biopolítico de los cuerpos. Se trata, sin dudas, de una lectura obligada del signo de nuestros tiempos. The X-Files nos viene a mostrar la fragilidad de la condición humana y la manifestación del poder soberano.

\section{Referencias bibliográficas}

Agamben, Giorgio (2013). "El gobierno de la inseguridad" en WAA. Pensar desde la izquierda. Mapa del pnesamiento crítico para un tiempo de crisis, pp. 25-35. Madrid: Errata Naturae.

Agamben, Giorgio (2010). Homo Sacer. El poder soberano y la nuda vida. Valencia: Pre-textos.

Agamben, Giorgio (2007). Estado de Excepción. Buenos Aires: Adriana Hidalgo editora.

Castro, Edgardo (2008). Giorgio Agamben. Una arquelogía de la potencia. Buenos Aires: Unsam Edita.

Eagleton, Terry (2006). La Estética como ideología. Madrid: Trotta.

Foucault, Michel (2009). Seguridad, Territorio, Población. Buenos Aires: FCE.

Foucault, Michel (2003). Vigilar y Castigar. Nacimiento de la prisión. Buenos Aires: Siglo XXI Editores.

Foucault, Michel (1992). Microfísica del poder. Madrid: Las Ediciones de La Piqueta.

Galera, Julieta y Nitrihual, Luis (2009). "Cantinflas, entre risas y sombras. Un análisis semiótico cínico". Anagramas, n. 8 (15), pp. 99-115.

Gómez, María del Carmen (2008). "La verdad están dentro de estas páginas. Las novelas de expediente X y los usos de la literatura de ciencia ficción". En línea: http://www.academia.edu/221730/_La_ verdad_esta_dentro_de_estas_paginas_Las_novelas_de_Expediente_X_y_los_usos_de_la_ literatura_de_ciencia_ficcion._Consultado el 6 de septiembre de 2013.

Gramsci, Antonio (1990). Cuadernos desde la carcel: Pasado y Presente. México: Juan Pablos Editor.

Jameson, Fredric (2009). Arqueologías del futuro. Madrid: AKAL.

Jameson, Fredric (1995). La estética geopolítica. Cine y espacio en el sistema mundial. Barcelona: Paidós.

Macho, Ramón (2012). "La estupidez de Fox Mulder: Gilles Deleuze y Expediente X: Breves notas paranormales acerca de la necedad" Revista de letras y ficción audiovisual, número 2, pp. 138 151.

Mosco, Vincent (2009). Renovando la Economía Política. Madrid: Bosch.

Nitrihual, Luis (2017). El asalto de la oligarquía. Medios de comunicación y sociedad neoliberal en Chile. Concepción: Escaparate.

Nitrihual, Luis; Galera Julieta (2011). "Romeo y Julieta de Cantinflas. El humor en la cultura de masas". Arte Individuo y Sociedad, n. ${ }^{\circ} 23$, Vol. 2. pp. 97-107.

Slotedijk, Peter (2007). Crítica de la razón cínica. Madrid: Siruela.

Sierra, Francisco (2006). Políticas de comunicación y educación. Crítica y desarrollo de la Sociedad de Conocimiento. Barcelona: Gedisa.

Thompson, John B. (1998). Los media y la modernidad. Una teoría de los medios de comunicación. Barcelona: Paidós 